\title{
Physician, Psychiatrist and Artificial Intelligence
}

\author{
Diyanath Samarasinghe
}

et's start by examining the question, 'How does a - physician differ from a psychiatrist?' This matter is more interesting to examine than the similarities, which are many and obvious. The foundations of psychiatric practice are, after all, in medicine is undisputed.

Let me list a few differences, from those I have heard. I use 'physician' here to mean all clinicians other than psychiatrists.

1. Patients and their families feel happier about a psychiatrist referring them to a physician than a physician referring them to a psychiatrist.

2. Psychiatrists feel they have a lower status than other clinical specialists.

3. Physicians limit their attention, more than do psychiatrists, to the disease alone.

4. Psychiatrists are generally vaguer than physicians.

5. It will take technological devices and 'Artificial Intelligence' longer to replace psychiatric practice than other forms of medical practice.

These may be true or simply a reiteration of stereotypes. I believe the last one- that trained humans will still be needed to provide psychiatry practice long after most of the interventions performed by ophthalmologists, obstetricians and cardiologists, for instance, are done by machines. I do worry that psychiatry practice as I see it is being gradually oversimplified to the point where it too can easily be replaced by a machine that provides correct prescriptions. The third on the list may simply be based on the public belief that psychiatrists are attuned to feelings, more than are other doctors.

\section{Taking stock \\ Patients may indeed expect psychiatrists to be special- ly caring and good at comforting them and easing their troubled minds. They may instead expect physicians to be efficient at identifying and treating the physical causes of their symptoms - that is, curing the disease. Some doctors too think that kindness and empathy are of secondary importance, a kind of nice but inessential component of management. Their number includes}

Honorary Professor in Psychiatry, Faculty of Medicine, University of Rajarata

Email:1dsamara@gmail.com

iD https://orcid.org/0000-0003-2891-8297 psychiatrists.

Let's try to rate ourselves on a scale from 0 to 10 on this factor. A ' 0 ' indicates we think that kindness, caring, empathy and warmth are not really relevant to good medical practice - even though they may be a pleasant accompaniment to it. A rating of 10 means we think that kindness and caring take clear precedence over efficiency in diagnosis and management. There clearly are different situations where one or the other extreme applies, but we should rate ourselves on our general tendency. An Al (Artificial Intelligence) machine, perfectly programmed, should give itself a rating of ' 0 ', since it will likely consider itself accurate on diagnosis and management which, presumably, does not rely on warmth and caring.

The self-rating exercise has practical implications for us human doctors. None of us will rate ourselves close to either extreme, even if we objectively belong there. But we may still recognize that we veer towards one pole. Our closeness to either end may be to a degree that requires corrective action. It will help to keep our self-rating in mind over the next few days, or years, and check if our perspective changes, on closer scrutiny.

\section{A re-evaluation}

In the 'psychiatrist versus physician' comparison, the opinion of the public is probably that psychiatrists are closer to the feeling pole, of caring and empathy, or that they should be. Physicians may generally be considered closer to the efficient diagnosis and management pole. Efficient diagnosis will likely be seen as nearer the Al or machine-deliverable practice.

This dichotomy between technically accurate management versus making the patient feeling cared for and happy may be misleading. In reality, the two are not ends of a spectrum but quite separate axes. The axes are not quite at right angles. One cannot really be a good diagnostician without the guidance provided to clinical judgement by genuine caring and empathy. The converse does not apply as much. One can be the tops in empathy but a terrible diagnostician.

The second item in our initial list was that psychiatrists feel that they are of a lower status than other clinical specialists. Not having studied this systematically, I cannot say whether this is any more likely than its opposite. But I have heard psychiatrists pontificate about 
not missing neurological disorders more than I have heard physicians declaring the need to avoid missing psychiatric disorders. This is not the only basis for my guess that psychiatrists are keener to be seen as clever physicians (basically, neurologists) than neurologists want to be seen as clever psychiatrists.

There are psychiatrists who insist that all psychiatric disorders are brain disease and nothing else. This formulation has some basis. But psychiatrists who claim this as an absolute should be careful about what that stance implies. A logical conclusion from it is that we then don't need a separate discipline called psychiatry. We should scrap the speciality called 'psychiatry' and absorb it into neurology. What basis is there for separating out a few brain diseases as the province of psychiatry? Does the separation of psychiatry from neurology persist only for historical reasons? If psychiatry is all brain disease, we should consider seriously whether we could reduce or stop new recruitments to psychiatry and let it steadily atrophy. The conditions treated by psychiatrists can gradually be absorbed by clinical medicine, mostly neurology.

\section{Commonalities}

The claim that psychiatrists are better able to handle emotional issues and more focussed than others on a 'whole-patient' outlook is probably false. These qualities, essential for all clinical work, are more likely to be distributed evenly among all doctors. Good doctoring demands that doctors, across specialities, are able to deliver what is most expected of us. Among these is that we effectively relieve pain, discomfort, disability and suffering. People come to us expecting relief from such things as difficulty in swallowing food, walking uphill, having intercourse, sleeping well, talking to strangers, breathing effortlessly and the like. It is probably correct to say that all of these entail some amount of suffering and that people are driven to consult us by their urge to reduce discomfort or suffering.

Not all forms of suffering are seen as subject matter for doctors, of course. Doctors are not consulted for the relief of suffering due to being robbed, cheated orvictimized by others, lack of enough money for food, a son being parasitic or the weather harsh. There is in society a fairly accurate perception of what kinds of suffering call for medical intervention. People may in error consult us for some matters that we can't deal with any better than a non-medical person; things about which we have no special expertise. We are not obliged to meet such misguided expectations. If a particular difficulty cannot be dealt with medically, it is up to us to re-direct those who consult us inappropriately.

\section{Suffering}

We may not recognize the many things we do, which contribute to relieving patients' suffering. In that event, we remain oblivious to the totality of what we deliver. When a suffering woman complains of burning pain in the upper abdomen, a limited model for understanding why she came to us is that it is purely because certain nerves are stimulated to carry afferent impulses to the brain due to an irritation or ulceration of the gastric mucosa. Accordingly, we see that our job is to treat the gastric lesion, which will reduce or stop these impulses and thereby relieve her suffering. But the relief she gets from seeing us occurs also through some other things we deliver, often unrecognized by us.

A physician treating GORD, or a psychiatrist treating a depressive episode in a person with bipolar disorder, may think that the physical basis of the patient's symptoms is all they treat. They both fail to recognize the wider stuff they deliver. They also miss out on the depth of what practicing medicine offers. One aspect of this depth is that our job offers us daily lessons on the causes and relief of human suffering.

\section{Relief}

Psychiatrists are probably no better than physicians, in understanding and relieving suffering. A patient who meets a surgeon is relieved of suffering, to different degrees, after the first consultation - and well before surgery that will deal with the bodily disorder. The extent of immediate relief is dependent on that surgeon's ability - often instinctive - to understand and relieve the patient's suffering. A consultation with this particular surgeon leads to much relief right away, despite the required surgery being scheduled for next month. There may be no such relief on consulting a different surgeon. The same applies to the immediate response to receiving a prescription from one particular psychiatrist rather than another.

It is good if all doctors can recognize this simple phenomenon and its basis. The easiest way to do so is to observe how patients' suffering responds to being dealt with by different doctors. Immediate feelings of relief are not due to some magical or secret gift that one doctor possesses and another does not. It is a quality that can be nurtured. This is not the place to take up in detail the subject of how we learn to do so. But we do have space to point to some underlying things that are useful to examine.

\section{A base for understanding}

We have been speaking of two elements here - suffering and pain (or physical discomfort). They overlap greatly, in the nature of their experience as well as in their ascribed meaning. We must try now to separate them. I believe that doing so will help us understand things better. Splitting pain from resultant suffering may be regarded as artificial or senseless. But let's do so anyway. 
In this separation, we can consider pain to be a purely physical or bodily occurrence, including how it is experienced. The experience of pain therefore requires an intact and conscious organism - a working brain in a working body. Suffering, on the other hand, is the distressing accompaniment of discomfort or disability. I think it is experienced at rather a different level. It is my contention that the experience of suffering requires the presence of a sufferer, beyond the working brain in a working body, a self. Our bodies experience pain. But it is we who suffer. Suffering due to causes other than pain is also the same. The sufferer is the person. The body that experiences pain is only the intact physical organism in which the person resides. The person is an abstraction, the self.

When we see doctoring only as ministration to the ailing body or organism, we miss the suffering person. It is the person who is relieved through our care, and caring. And she may begin to experience relief from the moment she sees you, the doctor. Or rather, from the moment you see her. And you often don't even know it. If you indeed don't, you have thus far missed part of the incredible reward that practicing medicine offers.

We must, at the same time, remember that all patients aren't equally sensitive or receptive to more subtle aspects of our interaction. But even the usually less sensitive tend to become more open and responsive when they are ill.

\section{The positive pole}

Examining the opposite end of the spectrum also offers many useful life lessons - namely, the relation between physical pleasure and the experience of joy or bliss. Medical practice does not normally provide stimuli to make us explore the connection between these two, rather nice, things. Our line of work exposes us mostly to pain and suffering.

The enlightened pursuit of pleasure and the experience of joy and bliss offer altogether different rewards. Enjoyment of life is at risk of being drowned in doctors' daily dealings with misery. There is of course scope for joy in routine medical practice, should we begin to notice the relief that we can and do provide for each patient's misery. If we had any sense, we'd strive unceasingly to become even more effective exponents of the art of relieving suffering. It offers us great potential for exhilaration, even in our day to day dealings with pain and misery.

It is good to remember that being human is defined by, among other things, the capacity to suffer. Even more so by the ability to relieve suffering. And most by the irrepressible urge to do so. It is our suffering that brings us together... We know that there is no help for us but from one another, that no hand will save us if we do not reach out our hand ....

All you have is what you are, and that you give. - Ursula le Guin 